\title{
Fuzzy single-period product problem with return policy*
}

\author{
Li Zhu, Ruiqing Zhao ${ }^{\dagger}$, Wansheng Tang \\ Institute of Systems Engineering, Tianjin University, Tianjin 300072, China
}

(Received February 25 2007, Accepted April 16 2007)

\begin{abstract}
This paper considers a single-period product problem with return policy of a supply chain composed by a supplier in the upper level and several retailers in the lower level. It's assumed that the supply chain is operated in a fuzzy environment. The fuzziness is associated with the market demand and goodwill cost per unit due to stock out. A fuzzy bilevel expected value model and a fuzzy chance-constrained model are formulated for this kind of problem. To solve the problem, by simulating the decision process a hybrid algorithm based on particle swarm optimizer (PSO), neural network and fuzzy simulation is developed. Finally, numerical examples are provided to illustrate the effectiveness of the algorithm.
\end{abstract}

Keywords: supply chain, fuzzy variables, bilevel model, hybrid algorithm

\section{Introduction}

Consider a supplier producing a single-period product and several retailers making orders from the supplier and then reselling the products to their final markets, respectively. Such is a classical newsboy problem, in which the objective is to find the retailers' order quantities maximizing their profits, respectively. This model is often used to make decision in modern industry to estimate the order quantity in advance.

During the past three decades, many literatures have focused on the newsboy problem. Lau and Lau ${ }^{[15]}$ studied it with price-dependent demand distribution. Shih ${ }^{[26]}$ considered the objective of maximizing the probability of attaining a profit target. Lau ${ }^{[16]}$ studied the reordering strategies for a single-period product. And Anvari $^{[1]}$ considered capital-asset-pricing-type objectives of newsboy-type inventory models . But most of these literatures considered the problem as a single level system from the view of the retailer, actually supplier and retailer interact with each other in a bilevel decision system. Recently, many literatures have considered the single-period product problem in a bilevel context, where both supplier and retailer are profit maximizers (risk neural). Iyer and Bergen ${ }^{[12]}$ presented formal models for the inventory decisions of supplier and retailer both before and after quick response. Pasternack ${ }^{[25]}$ showed that a policy offering partial credit to the retailer for all unsold items can achieve channel coordination.

Random variables are used to denote the uncertain parameters such as market demand in literatures. However, in many cases it is difficult to estimate the probabilities distribution of these parameters due to lack of historic data and it is reasonable for us to employ fuzzy variables to depict these parameters. The notion of fuzzy sets was originally introduced by Zadeh ${ }^{[27]}$ who initialized the concept of a fuzzy set via membership function and presented possibility measure to measure a fuzzy event ${ }^{[28]}$. Since then, Nahmias ${ }^{[23]}$, Kaufmann and Gupta ${ }^{[13]}$, Dubois and Prade ${ }^{[7]}$ and many other researchers did a plenty of research on possibility theory. The expected value of a fuzzy variable plays an important role in optimization problems with fuzziness. For the studies on the expected value operator of fuzzy variable, we can refer to Dubois and Prade ${ }^{[8]}$, Campos

\footnotetext{
* This work was supported by National Natural Science Foundation of China Grant No. 70571056 and Program for New Century Excellent Talents in University.

$\dagger$ E-mail address: zhao@tju.edu.cn.
} 
and González ${ }^{[4]}$, etc. Recently, Liu and $\mathrm{Liu}^{[21]}$ proposed a concept of credibility measure and and laid a new foundation for optimization theory in uncertain environments, in which fuzzy expected value models were proposed to deal with the optimization problems.

In this paper, we use fuzzy variables to depict the market demand and goodwill cost per unit due to stock out incurred by the retailer. Considering the supplier's returns policy proved to be effective by Padmanbhan ${ }^{\text {[24] }}$ and Emmons ${ }^{[9]}$ in supply chain coordination, this paper presents two fuzzy bilevel models based on the fuzzy expected value programming proposed by Liu and $\mathrm{Liu}^{[21]}$, and fuzzy chance-constrained programming by Liu and Iwamura ${ }^{[20]}$.

The remainder of this paper is organized as follows. Section 2 presents a brief description of the preliminary knowledge about fuzzy variables. Section 3 constructs a fuzzy bilevel expected model and a fuzzy bilevel chance-constrained model for the single-period product problem in a supply chain. Section 4 develops a hybrid algorithm based on particle swarm optimizer algorithm (PSO) neural network and fuzzy simulation to solve bilevel programming models. Some numerical examples are given in Section 5. Finally, we conclude this paper in Section 6.

\section{Fuzzy variables}

Let $\Theta$ be a nonempty set, and $P(\Theta)$ the power set of $\Theta$. In order to present the axiomatic definition of possibility, Nahmias ${ }^{[23]}$ and $\mathrm{Liu}^{[18]}$ give the following four axioms.

Axiom 1. $\operatorname{Pos}\{\Theta\}=1$.

Axiom 2. $\operatorname{Pos}\{\phi\}=0$, where $\phi$ denotes the empty set.

Axiom 3. $\operatorname{Pos}\left\{\bigcup_{i} A_{i}\right\}=\sup _{i} \operatorname{Pos}\left\{A_{i}\right\}$ for any collection $\left\{A_{i}\right\}$ in $P(\Theta)$.

Axiom 4. Let $\Theta_{i}$ be nonempty sets on which $\operatorname{Pos}_{i}\{\cdot\}$ satisfies the first three axioms, $i=1,2, \cdots, n$, respectively, and $\Theta=\Theta_{1} \times \Theta_{2} \times \cdots \times \Theta_{n}$. Then

$$
\operatorname{Pos}\{A\}=\sup _{\left(\theta_{1}, \theta_{2}, \cdots, \theta_{n}\right) \in A} \operatorname{Pos}_{1}\left\{\theta_{1}\right\} \wedge \operatorname{Pos}_{2}\left\{\theta_{2}\right\} \wedge \cdots \wedge \operatorname{Pos}_{n}\left\{\theta_{n}\right\}
$$

for each $A \in P(\Theta)$.

Definition 1. (Liu and Liu $^{[19]}$ ) Let $\Theta$ be a nonempty set, and $P(\Theta)$ the power set of $\Theta$. Then Pos is called a possibility measure if it satisfies the first three axioms, and $(\Theta, P(\Theta), P o s)$ is called a possibility space.

Remark 1. ( Liu $\left.^{[18]}\right)$ Suppose that $\left(\Theta_{i}, P\left(\Theta_{i}\right), P o s_{i}\right), i=1,2, \cdots, n$ are possibility spaces. By Axiom 4, $\left(\prod_{i=1}^{n} \Theta_{i}, P\left(\prod_{i=1}^{n} \Theta_{i}\right), \bigwedge_{i=1}^{n} \operatorname{Pos}_{i}\right)$ is also a possibility space, which is called the product possibility space.

Definition 2. (Nahmias $^{[23]}$ ) A fuzzy variable is defined as a function from a possibility space to the set of real numbers.

Definition 3. (Liu and Liu $^{[21]}$ ) Let $(\Theta, P(\Theta)$, Pos) be a possibility space, and $A$ a set in $P(\Theta)$. Then the credibility measure of $A$ is defined by

$$
\operatorname{Cr}\{A\}=\frac{1}{2}\left(\operatorname{Pos}\{A\}+1-\operatorname{Pos}\left\{A^{c}\right\}\right) .
$$

Definition 4. $\left(\right.$ Liu $\left.^{[19]}\right)$ A fuzzy variable $\xi$ is a function from the possibility space $(\Theta, \mathcal{P}(\Theta)$, Pos) to the set of real numbers, and its membership function is defined by

$$
\mu(x)=\operatorname{Pos}\{\theta \in \Theta \mid \xi(\theta)=x\}, \forall x \in R .
$$

Example 1. A trapezoidal fuzzy variable $\xi$ determined by quadruplet $\left(r_{1}, r_{2}, r_{3}, r_{4}\right)$ of crisp numbers with $r_{1}<r_{2}<r_{3}<r_{4}$, whose membership function is 


$$
\mu(x)=\left\{\begin{array}{cl}
\frac{x-r_{1}}{r_{2}-r_{1}}, & \text { if } r_{1} \leq x \leq r_{2} \\
1, & \text { if } r_{2} \leq x \leq r_{3} \\
\frac{x-r_{4}}{r_{3}-r_{4}}, & \text { if } r_{3} \leq x \leq r_{4} \\
0, & \text { otherwise. }
\end{array}\right.
$$

From the definition of credibility, it is easy to obtain

$$
\operatorname{Cr}\{\xi \leq x\}=\left\{\begin{array}{cl}
0, & \text { if } x \leq r_{1} \\
\frac{x-r_{1}}{2\left(r_{2}-r_{1}\right)}, & \text { if } r_{1} \leq x \leq r_{2} \\
\frac{1}{2}, & \text { if } r_{2} \leq x \leq r_{3} \\
\frac{x+r_{4}-2 r_{3}}{2\left(r_{4}-r_{3}\right)}, & \text { if } r_{3} \leq x \leq r_{4} \\
1, & \text { otherwise. }
\end{array}\right.
$$

Definition 5. (Liu and Liu ${ }^{[21]}$ ) Let $\xi$ be a fuzzy variable on the possibility space $(\Theta, \mathcal{P}(\Theta)$, Pos). The expected value $E[\xi]$ is defined by

$$
E[\xi]=\int_{0}^{+\infty} C r\{\xi \geq r\} \mathrm{d} r-\int_{-\infty}^{0} C r\{\xi \leq r\} \mathrm{d} r
$$

provided that at least one of the two integrals is finite. Especially, if the fuzzy variable $\xi$ is positive (i.e., $\operatorname{Pos}\{\xi \leq 0\}=0)$, then

$$
E[\xi]=\int_{0}^{+\infty} C r\{\xi \geq r\} \mathrm{d} r .
$$

Example 2. The expected value of a trapezoidal fuzzy variable $\xi=\left(r_{1}, r_{2}, r_{3}, r_{4}\right)$ is

$$
E[\xi]=\frac{1}{4}\left(r_{1}+r_{2}+r_{3}+r_{4}\right)
$$

Proposition 1. ( $\mathrm{Liu}$ and $\mathrm{Liu}^{[22]}$ ) Let $\xi_{1}$ and $\xi_{2}$ be two independent fuzzy variables with finite expected values. Then for any real numbers $a$ and $b$, we have

$$
E\left[a \xi_{1}+b \xi_{2}\right]=a E\left[\xi_{1}\right]+b E\left[\xi_{2}\right] .
$$

Definition 6. Let $\xi$ be a fuzzy variable, and $f: \Re \rightarrow \Re$ a function. Then the expected value of $f(\xi)$ is defined as

$$
E[f(\xi)]=\int_{0}^{+\infty} C r\{f(\xi) \geq r\} \mathrm{d} r-\int_{-\infty}^{0} C r\{f(\xi) \leq r\} \mathrm{d} r .
$$

\section{Fuzzy supply chain bilevel model}

In this section, without loss of generality, we discuss a common supply chain with one supplier and several retailers. The supplier produces an item of single-period products while each retailer makes an order from the supplier, then resell the products to his own market. The single-period product market is uncertain in real world, so that a certain retailer is likely to face two situations. One situation is that a certain retailer's inventory has been depleted by the end of the selling period, and the other is that the retailer keeps unsold products at the end of the selling period. The supplier offers a return policy to buy back the retailer's unsold products. To be more clearly, we formally list the notation and assumptions as follows. 


\subsection{Notation and assumptions}

\section{Notation}

$\Pi_{s i}$ supplier's partial profit function corresponding to the $i$ th retailer, $i=1,2, \cdots, n$

$\Pi_{s}$ supplier's total profit function

$\Pi_{r i} i$ th retailer's profit function

$s \quad$ supplier's production cost per unit

$v \quad$ return product's salvage value per unit of the supplier

$p \quad$ retail price per unit

$g_{i} \quad$ goodwill cost per unit due to stock out incurred by the $i$ th retailer, $i=1,2, \cdots, n$

$D_{i}$ market demand of the $i$ th retailer in a selling period, $i=1,2, \cdots, n$

$w$ supplier's wholesale price per unit, the supplier's decision variable

$b \quad$ supplier's buy back price per unit of unsold product, the supplier's decision variable

$q_{i} \quad i$ th retailer's order quantity, the $i$ th retailer's decision variable, $i=1,2, \cdots, n$

\section{Assumptions}

(1) Only one item product is considered in one selling period.

(2) The retailers are independent with each other.

(3) The supplier and all the retailers have full information about cost structures of each other.

(4) The market demand of the $i$ th retailer is assumed to be an independent fuzzy variable defined on the possibility space $\left(\Theta_{1_{i}}, P\left(\Theta_{1_{i}}\right), \operatorname{Pos}_{1_{i}}\right)$. The market demand especially for single-period product is often difficult to measure, and it is more suitable to be given a subjective assessment, so it is assumed to be a fuzzy variable $D_{i}$ and $i=1,2, \cdots, n$.

(5) The goodwill cost per unit due to stock out of the $i$ th retailer $g_{i}$ is assumed to be an independent fuzzy variable defined on the possibility space $\left(\Theta_{2_{i}}, P\left(\Theta_{2_{i}}\right), P o s_{2_{i}}\right)$. It is an uncertain parameter influenced by many factors and is difficult to obtain its precise value, so it is reasonable for us to denote it as a fuzzy variable $g_{i}$ and $i=1,2, \cdots, n$.

Considering the retailers' response, the supplier wishes to determine an optimal wholesale price $w$ and an optimal buy back price $b$ as joint decisions, and then the $i$ th retailer adjusts the order quantity $q_{i}$ according to the supplier's decisions to maximize his own profit. After that, the supplier seeks to maximize his profit function of $w, b, q_{i}$ and $i=1,2, \cdots, n$.

To the $i$ th retailer, he is likely to face two situations after the selling period, i.e., stockout $\left(D_{i}>q_{i}\right)$ and remain products unsold $\left(D_{i} \leq q_{i}\right)$. The profit function is given by

$$
\Pi_{r i}\left(w, b, q_{i}\right)= \begin{cases}p q_{i}-w q_{i}-g_{i}\left(D_{i}-q_{i}\right), & D_{i}>q_{i} \\ p D_{i}-w q_{i}+b\left(q_{i}-D_{i}\right), & D_{i} \leq q_{i}\end{cases}
$$

where in the case of stockout $\left(D_{i}>q_{i}\right), p q_{i}, w q_{i}$ and $g_{i}\left(D_{i}-q_{i}\right)$ are respectively the revenue, buying cost and the goodwill cost due to stock out of the $i$ th retailer, and in the case of remain products unsold $\left(D_{i} \leq q_{i}\right)$, $p D_{i}$ and $w q_{i}$ are the revenue and buying cost of the $i$ th retailer, and $b\left(q_{i}-D_{i}\right)$ is the buyback revenue from the supplier. Furthermore, the $i$ th retailer's profit function can be generally represented by

$$
\left.\Pi_{r i}\left(w, b, q_{1}, \cdots, q_{n}\right)=p \min \left\{q_{i}, D_{i}\right\}-w q_{i}+b \min \left\{q_{i}-D_{i}, 0\right\}\right)+g_{i} \max \left\{q_{i}-D_{i}, 0\right\} .
$$

The supplier's partial profit function corresponding to the $i$ th retailer, $i=1,2, \cdots, n$ is given by

$$
\Pi_{s i}\left(w, b, q_{i}\right)= \begin{cases}(w-s) q_{i}, & D_{i}>q_{i} \\ (w-s) q_{i}+(v-b)\left(q_{i}-D_{i}\right), & D_{i} \leq q_{i}\end{cases}
$$


in which $w q_{i}$ is the supplier's partial revenue given by the $i$ th retailer, $s q_{i}$ the supplier's partial production cost, and in the case of remain products unsold $\left(D_{i} \leq q_{i}\right), v\left(q_{i}-D_{i}\right)$ is the salvage revenue, $b\left(q_{i}-D_{i}\right)$ is the buy back cost. Furthermore, it can be generally written as

$$
\Pi_{s i}\left(w, b, q_{i}\right)=(w-s) q_{i}+(v-b) \max \left\{q_{i}-D_{i}, 0\right\} .
$$

The supplier's total profit function is given by

$$
\Pi_{s}\left(w, b, q_{1}, \cdots, q_{n}\right)=\sum_{i=1}^{n} \Pi_{s i}\left(w, b, q_{i}\right) .
$$

Since $D_{i}$ and $g_{i}$ are assumed to be fuzzy variables, $\Pi_{r i}\left(w, b, q_{i}\right), \Pi_{s i}\left(w, b, q_{i}\right)$ and $\Pi_{s}\left(w, b, q_{1}, \cdots, q_{n}\right)$ are fuzzy variables.

\subsection{Fuzzy bilevel expected value model}

In this problem, the interaction between the supplier and several retailers is viewed as a leader-follower game. Here, we depict this problem with bilevel programming which is a useful tool of studying decentralized decision system. In order to optimize the expected value of profit functions, we propose the following fuzzy bilevel expected value model (FBEVM),

$$
\begin{cases}\max _{w, b} & \mathrm{E}\left[\Pi_{s}\left(w, b, q_{1}, \cdots, q_{n}\right)\right] \\
\text { s.t. } & \\
& 0 \leq b \leq s \leq w \leq p \\
& q_{i} \text { solves the problem: } \\
& \left\{\begin{array}{r}
\max _{q_{i}} \mathrm{E}\left[\Pi_{r i}\left(w, b, q_{i}\right)\right] \\
\text { s.t. } \\
q_{i}>0, \text { integers } \\
i=1,2, \cdots, n .
\end{array}\right.\end{cases}
$$

Definition 7. A feasible array $\left(q_{1}^{*}, q_{2}^{*}, \cdots q_{n}^{*}\right)$ is called a retailers' rational reaction array with respect to the given $w, b$ by supplier, if $q_{i}^{*}$ satisfies that

$$
\mathrm{E}\left[\Pi_{r i}\left(w, b, q_{i}\right)\right] \leq \mathrm{E}\left[\Pi_{r i}\left(w, b, q_{i}^{*}\right)\right]
$$

for any feasible $q_{i}, i=1,2, \cdots, n$.

Definition 8. A feasible array $\left(w^{*}, b^{*}, q_{1}^{*}, q_{2}^{*}, \cdots, q_{n}^{*}\right)$ is called a Stackelberg equilibrium of FBEVM if it satisfies that

$$
\mathrm{E}\left[\Pi_{s}\left(\bar{w}, \bar{b}, \overline{q_{1}}, \overline{q_{2}}, \cdots, \overline{q_{n}}\right)\right] \leq \mathrm{E}\left[\Pi_{s}\left(w^{*}, b^{*}, q_{1}^{*}, q_{2}^{*}, \cdots q_{n}^{*}\right)\right],
$$

for any $\bar{w}, \bar{b}$ and the retailers' rational reaction array $\left(\overline{q_{1}}, \overline{q_{2}}, \cdots, \overline{q_{n}}\right)$ with respect to $\bar{w}, \bar{b}$.

All retailers with equal status have to reveal their decisions simultaneously. The retailers' rational reaction array means that any retailer cannot improve his own profit by altering his decision. And the Stackelberg equilibrium is the solution of this leader-follower game. 


\subsection{Fuzzy bilevel chance-constrained model}

Chance-constrained programming was initialized by Charnes and Cooper ${ }^{[6]}$, and then was extended to the fuzzy decision systems by Liu and Iwamura ${ }^{[20]}$. The essential idea of chance-constrained programming is to optimize the optimistic return with a given confidence level subject to some chance constraints. Now we present a fuzzy bilevel chance-constrained programming model to describe the supply chain problem.

Chance constraint means that the constraint will hold at a confidence level provided as an appropriate safety margin by the decision-maker. The supplier provides a confidence level $\alpha_{0}$ at which it is desired the fuzzy event

$$
\Pi_{s}\left(w, b, q_{1}, \cdots, q_{n}\right) \geq f_{s}
$$

holds, where $f_{s}$ is a prospective value. Then the supplier's objective is to maximize the critical value $f_{s}$ with a chance constraint as follows,

$$
\operatorname{Cr}\left\{\Pi_{s}\left(w, b, q_{1}, \cdots, q_{n}\right) \geq f_{s}\right\} \geq \alpha_{0} .
$$

Then we turn attention to the $i$ th retailer who concerns about maximizing the critical value $f_{r i}$ with a chance constraints as follows,

$$
\operatorname{Cr}\left\{\Pi_{r i}\left(w, b, q_{i}\right) \geq f_{r i}\right\} \geq \alpha_{i},
$$

where $\alpha_{i}$ is the confidence level provided by $i$ th retailer, $i=1,2, \cdots, n$.

In order to maximize the critical values of the supplier and retailers, we have the following fuzzy bilevel chance-constrained model (FBCCM),

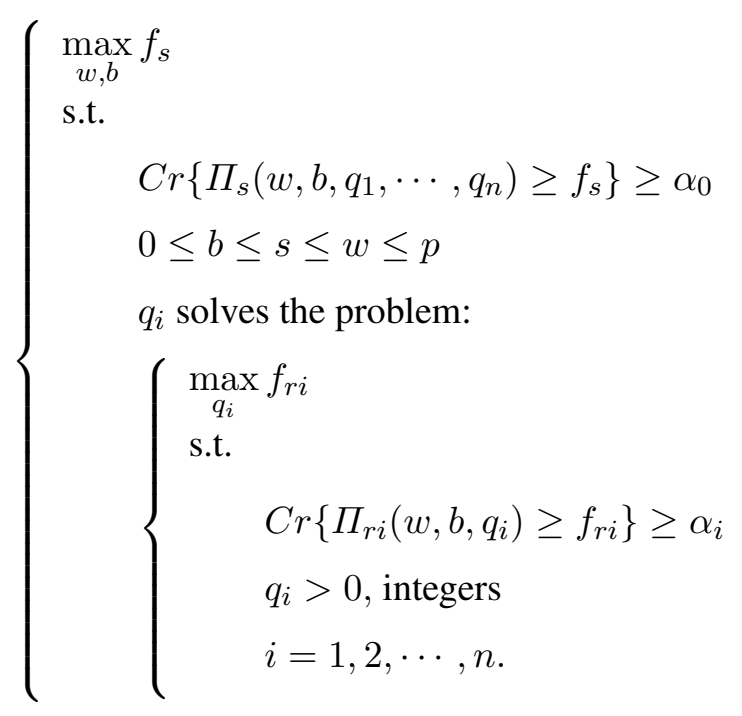

Definition 9. A feasible array $\left(q_{1}^{*}, q_{2}^{*}, \cdots, q_{n}^{*}\right)$ is called a retailers' rational reaction array with respect to the given $w$, b by supplier, if $q_{i}^{*}$ satisfies that

$$
\max \left\{f_{r i} \mid \operatorname{Cr}\left\{\Pi_{r i}\left(w, b, q_{i}\right) \geq f_{r i}\right\} \geq \alpha_{i}\right\} \leq \max \left\{f_{r i} \mid \operatorname{Cr}\left\{\Pi_{r i}\left(w, b, q_{i}^{*}\right) \geq f_{r i}\right\} \geq \alpha_{i}\right\},
$$

for any feasible $\left(q_{1}^{*}, q_{2}^{*}, \cdots, q_{i-1}^{*}, q_{i}, q_{i+1}^{*}, \cdots, q_{n}^{*}\right), i=1,2, \cdots, n$.

Definition 10. A feasible array $\left(w^{*}, b^{*}, q_{1}^{*}, q_{2}^{*}, \cdots q_{n}^{*}\right)$ is called a Stackelberg equilibrium of FBCCM if it satisfies that

$\max \left\{f_{s} \mid \operatorname{Cr}\left\{\Pi_{s}\left(\bar{w}, \bar{b}, \overline{q_{1}}, \overline{q_{2}}, \cdots, \overline{q_{n}}\right) \geq f_{s}\right\} \geq \alpha_{0}\right\} \leq \max \left\{f_{s} \mid \operatorname{Cr}\left\{\Pi_{s}\left(w^{*}, b^{*}, q_{1}^{*}, q_{2}^{*}, \cdots, q_{n}^{*}\right) \geq f_{s}\right\} \geq \alpha_{0}\right\}$,

for any $\bar{w}, \bar{b}$ and the retailers' rational reaction array $\left(\overline{q_{1}}, \overline{q_{2}}, \cdots, \overline{q_{n}}\right)$ with respect to $\bar{w}, \bar{b}$. 
Remark 2. If the fuzzy variables degenerate into crisp numbers, the models (10) and (16) can degenerate into the following bilevel supply chain model

$$
\begin{cases}\max _{w, b} & \Pi_{s}\left(w, b, q_{1}, \cdots, q_{n}\right) \\
\text { s.t. } & \\
& 0 \leq b \leq s \leq w \leq p \\
& q_{i} \text { solves the problem: } \\
& \left\{\begin{array}{c}
\max _{q_{i}} \Pi_{r i}\left(w, b, q_{i}\right) \\
\text { s.t. } \\
\\
\end{array} \quad \begin{array}{l}
q_{i}>0, \text { integers } \\
i=1,2, \cdots, n .
\end{array}\right.\end{cases}
$$

Remark 3. If the market demand $D_{i}$ and goodwill cost per unit due to stock out $g_{i}$ of the $i$ th retailer degenerate into crisp numbers, then the retailers' rational array is the array $\left(q_{1}^{*}, q_{2}^{*}, \cdots, q_{n}^{*}\right)$ with respect to $w, b$ given by supplier, if $q_{i}^{*}$ satisfies that $\Pi_{r i}\left(w, b, q_{i}\right) \leq \Pi_{r i}\left(w, b, q_{i}^{*}\right)$, for any feasible $q_{i}, i=1,2, \cdots, n$. And a Stackelberg equilibrium of model (19) is the array $\left(w^{*}, b^{*}, q_{1}^{*}, q_{2}^{*}, \cdots, q_{n}^{*}\right)$ satisfying $\Pi_{s}\left(\bar{w}, \bar{b}, \overline{q_{1}}, \overline{q_{2}}, \cdots, \overline{q_{n}}\right) \leq$ $\Pi_{s}\left(w^{*}, b^{*}, q_{1}^{*}, q_{2}^{*}, \cdots, q_{n}^{*}\right)$, for any $\bar{w}, \bar{b}$ and the retailers' rational reaction array $\left(\overline{q_{1}}, \overline{q_{2}}, \cdots, \overline{q_{n}}\right)$ with respect to $\bar{w}, \bar{b}$.

\section{Hybrid algorithm}

Ben-Ayed and Blair ${ }^{[2]}$ pointed out that bilevel programming is an NP-hard problem via the wellknown Knapsack Problem. In the past years, many numerical algorithms have been designed to solve bilevel

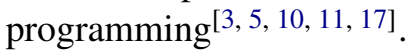

Now we design a hybrid algorithm based on fuzzy simulation, neural network and hierarchical particle swarm optimizer algorithm (PSO) to solve bilevel programming models, where PSO is employed to find the optimal result of the model.

\subsection{Fuzzy simulation}

In FBEVM, the objective functions of the supplier and the retailers are expected values of fuzzy variables, and in FBCCM we face the problem to find the maximal $f_{s}$ and $f_{r i}$. Since it is difficult to obtain these values by an analytic method, we resort to fuzzy simulation technique proposed by $\mathrm{Liu}^{[19]}$ to compute these values.

Here we put forward the procedure to estimate the value of $\mathrm{E}\left[\Pi_{r i}\left(w, b, q_{i}\right)\right]$, and the similar procedure can be used to obtain the values of $\mathrm{E}\left[\Pi_{s}\left(w, b, q_{1}, \cdots, q_{n}\right)\right]$. According to definition 6 , we have

$$
\mathrm{E}\left[\Pi_{r i}\left(w, b, q_{i}\right)\right]=\int_{0}^{+\infty} C r\left\{\Pi_{r i}\left(w, b, q_{i}\right) \geq r\right\} \mathrm{d} r-\int_{-\infty}^{0} C r\left\{\Pi_{r i}\left(w, b, q_{i}\right) \leq r\right\} \mathrm{d} r .
$$

The procedure is described as follows.

Step 1. Initialize parameters $p, v, s$, and set $\mathrm{E}\left[\Pi_{r i}\right]=0$.

Step 2. Define $M$ as the maximum number of iterations in random fuzzy simulation where $M$ is sufficiently large number. Randomly generate $\theta_{k}$ from $\Theta$, respectively, such that $\operatorname{Pos}\left\{\theta_{k}\right\} \geq \varepsilon$, where $k=1,2, \cdots, M$, and $\varepsilon$ is a sufficiently small positive number.

Step 3. Set $l=\Pi_{r}\left(\theta_{1}\right) \wedge \Pi_{r}\left(\theta_{2}\right) \wedge \cdots \wedge \Pi_{r}\left(\theta_{M}\right)$ and $u=\Pi_{r}\left(\theta_{1}\right) \vee \Pi_{r}\left(\theta_{2}\right) \vee \cdots \vee \Pi_{r}\left(\theta_{M}\right)$.

Step 4. Set $v_{k}=\operatorname{Pos}\left\{\theta_{k}\right\}$.

Step 5. Randomly generate $r$ from $[l, u]$. 
Step 6. If $r \geq 0$, then $\mathrm{E}\left[\Pi_{r i}\right] \leftarrow \mathrm{E}\left[\Pi_{r i}\right]+C r\left\{\Pi_{r i}\left(\theta_{k}\right) \geq r\right\}$, where the credibility $C r\left\{\Pi_{r i}\left(\theta_{k}\right) \geq r\right\}$ can be estimated by

$$
\frac{1}{2}\left(\max _{1 \leq k \leq M}\left\{v_{k} \mid \Pi_{r i}\left(\theta_{k}\right) \geq r\right\}+\min _{1 \leq k \leq M}\left\{1-v_{k} \mid \Pi_{r i}\left(\theta_{k}\right)<r\right\}\right) .
$$

Step 7. If $r<0$, then $\mathrm{E}\left[\Pi_{r i}\right] \leftarrow \mathrm{E}\left[\Pi_{r i}\right]-C r\left\{\Pi_{r i}\left(\theta_{k}\right) \leq r\right\}$, where the credibility $\operatorname{Cr}\left\{\Pi_{r i}\left(\theta_{k}\right) \leq r\right\}$ can be estimated by

$$
\frac{1}{2}\left(\max _{1 \leq k \leq M}\left\{v_{k} \mid \Pi_{r i}\left(\theta_{k}\right) \leq r\right\}+\min _{1 \leq k \leq M}\left\{1-v_{k} \mid \Pi_{r i}\left(\theta_{k}\right)>r\right\}\right) .
$$

Step 8. Repeat the 4 th to 6 th steps for $M$ times.

Step 9. $\mathrm{E}\left[\Pi_{r i}\left(w, b, q_{i}\right)\right]=l \vee 0+u \wedge 0+\mathrm{E}\left[\Pi_{r i}\left(w, b, q_{i}\right)\right] \frac{l-u}{M}$.

In FBCCM, we design a fuzzy simulation to find the maximal $f_{s}$ and $f_{r i}$ according to $\mathrm{Liu}^{[19]}$. If the value of $C r\left\{\Pi_{r i}\left(w, b, q_{i}\right) \geq f_{r i}\right\}$ can be obtained, the value of $C r\left\{\Pi_{s}\left(w, b, q_{1}, \cdots, q_{n}\right) \geq f_{s}\right\}$ can be acquired in a similar way. Then the model can be solved consequently. Here we design the fuzzy simulation to estimate $\operatorname{Cr}\left\{\Pi_{r i}\left(w, b, q_{i}\right) \geq f_{r i}\right\}$.

We randomly generate $\theta_{k}$ from $\Theta$ such that $\operatorname{Pos}\left\{\theta_{k}\right\} \geq \varepsilon$, and write $v_{k}=\operatorname{Pos}\left\{\theta_{k}\right\}, k=1,2, \cdots, M$, where $\varepsilon$ is a sufficiently small positive number. For any number $r$, we set

$$
L(r)=\frac{1}{2}\left(\max _{1 \leq k \leq M}\left\{v_{k} \mid \Pi_{s}\left(\theta_{k}\right) \geq r\right\}+\min _{1 \leq k \leq M}\left\{1-v_{k} \mid \Pi_{s}\left(\theta_{k}\right)<r\right\}\right) .
$$

It follows from monotonicity that we may employ bisection search to the maximal value $r$ such that $L(r) \geq \alpha$. This value is an estimation of $f_{r i}$. We summarize this process as follows.

Step 1. Randomly generate $\theta_{k}$ from $\Theta$ such that $\operatorname{Pos}\left\{\theta_{k}\right\} \geq \varepsilon$, for $k=1,2, \cdots, M$, respectively, where $\varepsilon$ is a sufficiently small positive number, $M$ is sufficiently large number.

Step 2. Find the maximal value $r$ such that $L(r) \geq \alpha$ holds.

Step 3. Return $r$.

\subsection{Neural network based on fuzzy simulation}

Neural network (NN) is a computing technique that imitates the cerebral structure and function. It is essentially a nonlinear mapping from the input space to the output space. It is heavily used in pattern recognition, uncertain function approximation, intelligent control and so on. One of its advantages is that it has high speed operation after it is well trained.

In this paper we choose the popular back-propagation (BP) neural network to simulate the objective functions, i.e., $\mathrm{E}\left[\Pi_{s}\left(w, b, q_{1}, \cdots, q_{n}\right)\right], \operatorname{Cr}\left\{\Pi_{r i}\left(w, b, q_{i}\right) \geq f_{r i}\right\}$, etc. To train an $\mathrm{NN}$, we first generate a certain number of groups of input data, including the supplier's decisions and the retailer's decisions, and then use fuzzy simulation to calculate the output data for each group of input data. After standardizing the input and output data, we train data with BP neural network to get the weights which can be used to evaluate the objection functions.

In the hybrid algorithm, we train two types of neural networks based on fuzzy simulation. One is $\mathrm{NN}-u$ used in the upper level, and the other is NN-l used in the lower level. Much computing time can be saved in the solution process by employing NN. For detailed discussion, the reader may consult ${ }^{[19]}$.

\subsection{Pso for bilevel programming}

The particle swarm optimizer (PSO) inspired by the behavior of animals such as bird flocking is a population-based algorithm that was invented by Kennedy and Eberhart ${ }^{[14]}$. It has been applied to a variety of domains such as game learning, data clustering, image analysis, function optimization and NN training. We employ PSO in this paper to solve the models of FBEVM and FBCCM. 
PSO is based on an n-dimensional swarm of particles, each of which indicates a possible solution of the problem space. The evolution can be described as such a procedure that each particle with memory of its best position will change its velocity in a specific direction so as to search for a better position amongst all the particles. The mathematical description is as follows,

$$
\begin{aligned}
& v_{i d}=\tilde{w} v_{i d}+c_{1} r_{1}\left(P_{i d}-x_{i j}\right)+c_{2} r_{2}\left(P_{g d}-x_{i d}\right) \\
& x_{i d}=x_{i d}+v_{i d},
\end{aligned}
$$

in which $x_{i d}$ and $v_{i d}$ represent the position and the velocity of particle $i$ in dimension $d, P_{i d}$ and $P_{g d}$ represent the personal best position and the global best position of particle $i$ in dimension $d, c_{1}$ and $c_{2}$ represent the acceleration coefficients and usually $c_{1}=c_{2}=2, r_{1} \sim U(0,1)^{n}$ and $r_{2} \sim U(0,1)^{n}$ are random coefficients, and $\tilde{w}$ is inertia weight.

In this paper, the models we have to solve are bilevel, and it is difficult to solve them with general PSO. In order to deal with this kind of problem, we design a bilevel PSO which employs PSO- $u$ and PSO- $l$ to settle upper level and lower level of the model, respectively.

After the upper level supplier gives the value of $w, b$, in order to to find the maximum profit of the lower level retailers, we give the following algorithm.

Step 1. Train NN-l based on fuzzy simulation.

Step 2. Initialize the initial velocity and position of each particle of PSO-l in lower level.

Step 3. Calculate the fitness of particle $i$ of PSO- $l$ with NN- $l$.

Step 4. Compare the value received in Step 3 with the personal best position particle $i$ of PSO- $l$ has arrived at. If the new value is better, make it the best position of particle $i$.

Step 5. Compare the value received in Step 3 with the global best position all particle of PSO- $l$ have arrived at. If the new value is better, make it the best position of particle $i$.

Step 6. Evolve the velocity and position of particle $i$ belonging to PSO- $l$ by (21), (22).

Step 7. Return to Step 3 if a sufficient good result hasn't been gotten or a predetermined circulation time hasn't been met.

Step 8. Return the best particle of lower level to the upper level.

In this paper, in order to solve the bilevel models, we present a hierarchical PSO algorithm which employs PSO- $u$ and PSO- $l$ to solve the upper level and lower level, respectively. The procedure of the hybrid algorithm based on fuzzy simulation, neural network and PSO is shown in the Fig. 1. model.

By a similar process, we can also design an algorithm to solve the fuzzy bilevel chance-constrained

\section{Numerical examples}

In this section, we will give some numerical examples to illustrate that the proposed algorithm is applicable and effective to solve the proposed models.

Example 3. Consider a supply chain, where a supplier is in upper level and three independent retailers are in lower level. The supplier offers a return policy to buy back the retailers' unsold products. The market demands and goodwill costs of three retailers are fuzzy variables, which are shown in Tab. 1. The other parameters are given as follows: $p=60, s=35, v=25$.

Table 1. Fuzzy demands and goodwill costs due to stockout

\begin{tabular}{c|c|c|c}
\hline$i$ & retailer 1 & retailer 2 & retailer 3 \\
\hline$g_{i}$ & $(1,2,3)$ & $(0.5,2,3.5)$ & $(2,3,4)$ \\
\hline$D_{i}$ & $(50,55,60)$ & $(70,80,85)$ & $(100,120,130)$ \\
\hline
\end{tabular}

If the supplier and retailers want to maximize their expected values of profit functions, we may use FBEVM to solve the problem. When the parameters in PSO- $u$ and PSO- $l$ are set as follows: the number of 


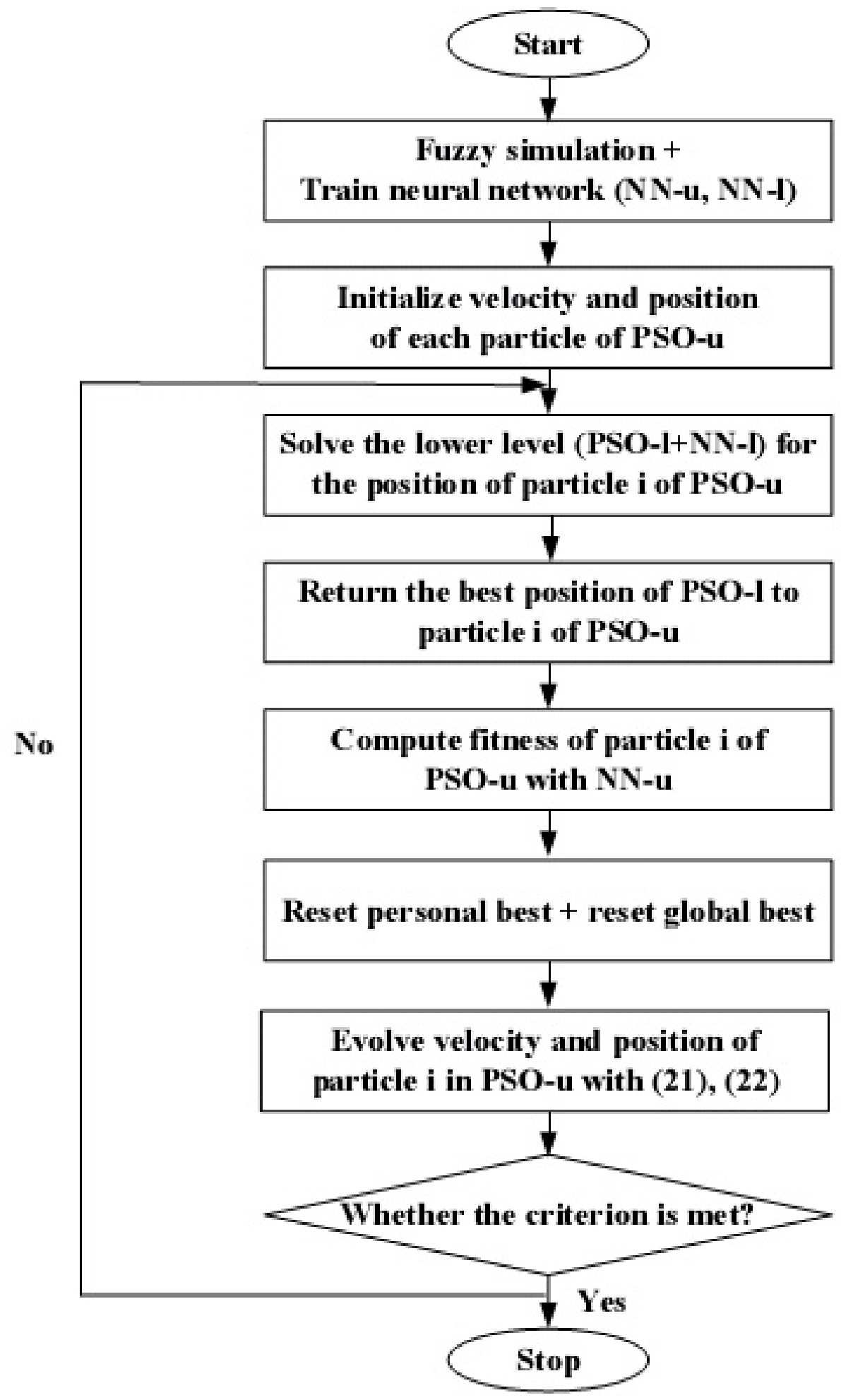

Fig. 1. The procedure of the algorithm

particles is 40 , the acceleration coefficiens $c_{1}$ and $c_{2}$ are set to 2 , the inertia weight $\bar{w}$ is set to 1 . The hybrid algorithm has been run with 5000 cycles in fuzzy simulation, 3000 samples in training neural networks, and 9000 generations in PSO. The result is shown in Tab. 2. We can get the Stackelberg equilibrium of FBEVM is

$$
\left(w, b, q_{1}, q_{2}, q_{3}\right)=(49.3,28.4,53,83,117) .
$$

The change of the expected values is shown in Fig. 2, from which it is obvious that the expected profit values 
Table 2. The result of FBEVM in Example 3

\begin{tabular}{c|c|c|c|c|c|c|c|c}
\hline \multicolumn{3}{c|}{ supplier } & \multicolumn{2}{c|}{ retailer 1 } & \multicolumn{2}{c|}{ retailer 2 } & \multicolumn{2}{c}{ retailer 3 } \\
\hline$E\left[\Pi_{s}\right]$ & $w$ & $b$ & $E\left[\Pi_{r 1}\right]$ & $q_{1}$ & $E\left[\Pi_{r 2}\right]$ & $q_{2}$ & $E\left[\Pi_{r 3}\right]$ & $q_{3}$ \\
\hline 3717.1 & 49.3 & 28.4 & 597.3 & 56 & 750.1 & 83 & 1137.9 & 117 \\
\hline
\end{tabular}

of the supplier and retailers approach to be steady after 3000 generation. Then we can say that the hybrid algorithm used to solve the numerical example is effective.



Fig. 2. The expected values of supplier and retailers with different iterations

Example 4. Consider the same supply chain in Example 3. If the supplier wants to obtain a prospective value with a property safety margin 0.8 , and the three retailers hope to get their own critical values with predetermined confidence levels $0.9,0.9,0.9$, respectively. The fuzzy demands and goodwill costs per unit due to stockout and other parameters are the same as Example 3. We may use FBCCM to solve the problem.

After a run of the hybrid algorithm with 5000 cycles in fuzzy simulation, 3000 samples in training neural networks, and 9000 generations in PSO, we get the result shown in Tab. 3. And the Stackelberg equilibrium of $\mathrm{FBCCM}$ is

$$
\left(w, b, q_{1}, q_{2}, q_{3}\right)=(47.2,30.1,57,78,114) .
$$

Table 3. The result of FBCCM in Example 4

\begin{tabular}{c|c|c|c|c|c|c|c|c}
\hline \multicolumn{3}{c|}{ supplier } & \multicolumn{2}{c|}{ retailer 1 } & \multicolumn{2}{c|}{ retailer 2 } & \multicolumn{2}{c}{ retailer 3 } \\
\hline$f_{s}$ & $w$ & $b$ & $f_{r 1}$ & $q_{1}$ & $f_{r 2}$ & $q_{2}$ & $f_{r 3}$ & $q_{3}$ \\
\hline 3027.6 & 47.2 & 30.1 & 669.8 & 57 & 998.4 & 78 & 1456.2 & 114 \\
\hline
\end{tabular}

\section{Conclusion}

This paper characterizes the market demand and goodwill cost per unit due to stock out as fuzzy variables. Considering the supplier's return policy in a single-period product problem, we propose a fuzzy bilevel 
expected value model and a fuzzy bilevel chance-constrained model. In order to solve the models, a hybrid algorithm is designed by integrating PSO, neural network and fuzzy simulation. Two numerical examples show that the hybrid algorithm is effective and robust.

\section{References}

[1] M. Anvari. Optimality criteria and risk in inventory models: The case of the newsboy problem, Journal of The Oprational Research Society, 1987, 38: 625-632.

[2] O. Ben-Ayed, C. Blair. Computional difficulties of bilevel linear programming, Operations Research, 1990, 38: 556-560.

[3] W. Bialas, M. Karwan. Two-level linear programming, Management Science, 1984, 30: 1004-1020.

[4] L. Campos, A. González. A subjective approach for ranking fuzzy numbers, Fuzzy Sets and Systems, 1989, 29: 145-153.

[5] W. Candler, R. Townaley. A linear two-level programming problem, Computer and Operations Research, 1982, 9: 59-76.

[6] A. Charnes, W. Cooper. Chance-constrained programming, Management Science, 1959, 6: 73-79.

[7] D. Dubois, H. Prade. Possibility Theory: An Approch to Computerized Processing of Uncertainty, Plenum, New York, 1988.

[8] D. Dubois, H. Prade. The mean value of a fuzzy number, Fuzzy Sets and Systems, 1987, 24: 279-300.

[9] H. Emmons, S. Gilbert. The role of returns policies in pricing and inventory decisions for catalogue goods, Management Science, 1998, 44: 276-283.

[10] J. Gao, B. Liu. Fuzzy multilevel programming with a hybrid intelligent algorithm, Computers and Mathematics with Applications, 2005, 49: 1539-1548.

[11] J. Gao, B. Liu, M. Gen. A hybrid intelligent algorithm for stochastic multilevel programming, IEEJ Trans. EIS, 2004, 124: 1-8.

[12] A. Iyer, M. Bergen. Quick respones in manufacturer-retailer channels, Management Science, 1997, 43: 59-70.

[13] A. Kaufmann, M. Gupta. Introduction to Fuzzy Arithmetic: Theory and Applications, Van Nostrand Reinhold, New York, 1985.

[14] J. Kennedy, R. Eberhart. Particle swarm optimization, IEEE International Conference on Neural Networks, Conference Proceedings, 1995, 4: 1942-1948.

[15] A. Lau, H. Lau. The newsboy problem with price-dependent demand distribution, IIE Transactions, 1988, 20: 168-175.

[16] H. Lau, A. Lau. Reordering strategies for a newsboy-type product, European Journal of Operational Research, 1997, 103: 557-572.

[17] B. Liu. Stackelberg-Nash equilibrium for multi-level programming with multiple follower using genetic algorithm, Computers Math. Applic, 1998, 36: 79-89.

[18] B. Liu. Toward fuzzy optimization without mathematical ambiguity, Fuzzy Optimization and Decision Making, 2002, 1: 43-63.

[19] B. Liu. Theory and Practice of Uncertain Programming, Phisica-Verlag, Heidelberg, 2002.

[20] B. Liu, K. Iwammura. Chance constrained programming with fuzzy parameters, Fuzzy Sets and Systems, 1998, 94: 227-237.

[21] B. Liu, Y. Liu. Excepted value of fuzzy variable and fuzzy expected value models, IEEE Transactions on Fuzzy Systems, 2002, 10: 445-450.

[22] Y. Liu, B. Liu. Expected value operator of random fuzzy variable and random fuzzy expexted values, International Journal of Uncertainty, Fuzziness \& Konwledged-Based Systems, 2003, 11: 195-215.

[23] S. Nahmias. Fuzzy variables, Fuzzy Sets and Systems, 1978, 1: 97-110.

[24] V. Padmanbhan, I. Png. Returns policies: Make money by making good, Sloan Management Review, 2002, 37: 65-72.

[25] B. Pasternack. Optimal pricing and return policies for perishable commodities, Marketing Science, 1985, 4: 166176.

[26] W. Shih. A general decision model for cost-volume-profit analysis under uncertainty, The Accounting Review, 1979, 54: $687-706$.

[27] L. Zadeh. Fuzzy sets, Information and Control, 1965, 8: 338-353.

[28] L. Zadeh. Fuzzy sets as a basis for a theory of possibility, Fuzzy Sets and Systems, 1978, 1: 3-28. 